\title{
Experimental Characterization of Biosensor based on Surface Plasmon Nano Interferometer
}

\author{
Peter Debackere, Peter Bienstman, Roel Baets \\ Photonics Research Group \\ Department of Information Technology, Ghent University - Interuniversity Micro-Electronics Center \\ Sint-Pietersnieuwstraat 41, Ghent, Belgium \\ Email: Peter.Debackere@intec.UGent.be
}

\begin{abstract}
Theoretically, label-free biosensing can be achieved by a Surface Plasmon Interferometer in Silicon-on-Insulator. We present in this paper measurement results validating this theoretical prediction and show this device to be capable of bulk refractive index sensing.
\end{abstract}

\section{INTRODUCTION}

The combination of Surface Plasmon interrogation techniques and Silicon-on-Insulator as a host substrate is a promising candidate for label-free biosensing [2], [5]. Due to the very strong evanescent fields near the interface Surface Plasmons promise to be very sensitive to refractive index changes, the gold layer boasts the advantage that it is a bio-compatible material and a very mature thiol-chemistry can be used for the functionalisation of this layer. The nanophotonics Siliconon-Insulator platform has the advantage that devices can be fabricated on a wafer scale using standard CMOS processing, allowing for cheap mass fabrication, integration with electronics, thus paving the route for real Lab-on-Chip applications. The high-index contrast between the silicon and silica allows to dramatically reduce the size of devices using nanophotonic waveguides [3].

A Surface Plasmon Interferometer combines the best of both worlds. The principle is based on the interference of two decoupled Surface Plasmon modes propagating on either side of a thin metallic layer embedded into a Silicon waveguide [5]. Theoretical results predict that this device would be capable of detecting very small refractive index changes $\left(10^{-6} R I U\right)$ for a device which has a physical footprint of only $100 \mu \mathrm{m}^{2}$. The transduction principle which is the basis for this device (Surface Plasmon Interference) has, up till now, not been verified experimentally.

\section{FABRICATION}

In order to validate our theoretical predictions a sample containing several Surface Plasmon Interference structures was fabricated. The sample features devices with a large array of parameters. The length is varied from 4 to $10 \mu \mathrm{m}$, in steps of 1 $\mu \mathrm{m}$. Waveguide widths are 10,3 and $0.550 \mu \mathrm{m}$. The layer stack consists of a Silicon substrate, $2 \mu \mathrm{m}$ of HDP-Silicon dioxide and $220 \mathrm{~nm}$ of a-Si, deposited using a PECVD process with Silane and He gases [6]. The devices were fabricated using Deep-UV Lithography in IMEC. Etch depth of the sensor region in the Si was $70 \mathrm{~nm}$, the $A u$ layer was deposited using thermal evaporation and has a thickness of $37 \mathrm{~nm}$.

\section{Proof-OF-Principle : MEASUREMENT}

Light from a single mode fiber was coupled into the sample using a lensed fiber, out coupling occurs through an objective. Measurements have been performed using a tunable laser and power detector, to ensure that only TM polarized light is measured a polarizer has been used in front of the detector. Although the devices were designed to be used in an aqueous environment, samples were measured in contact with air in order to obtain proof-of-principle.

Measurement results for this sample are depicted in figures 1, 2 and 3. The measured spectra are for TM-polarized light and they are normalised to a reference photonic broad (10 $\mu \mathrm{m})$ waveguide. While more measurements results have been obtained than the ones shown here (e.g. 4, 6, 7 and $9 \mu \mathrm{m}$ long devices were also characterised), the main conclusions can be drawn from studying these three measurement spectra. Absolute mean deviation error bars represent the fact that for each length, several identical devices have been measured. The red curves depict the results obtained for $10 \mu \mathrm{m}$ broad waveguides, blue curves depict results obtained for $3 \mu \mathrm{m}$ waveguides. For comparison, theoretical results are also depicted in the same graphs in green. Theoretical curves were calculated using CAMFR, an in-house developed eigenmode expansion tool [1], [4].

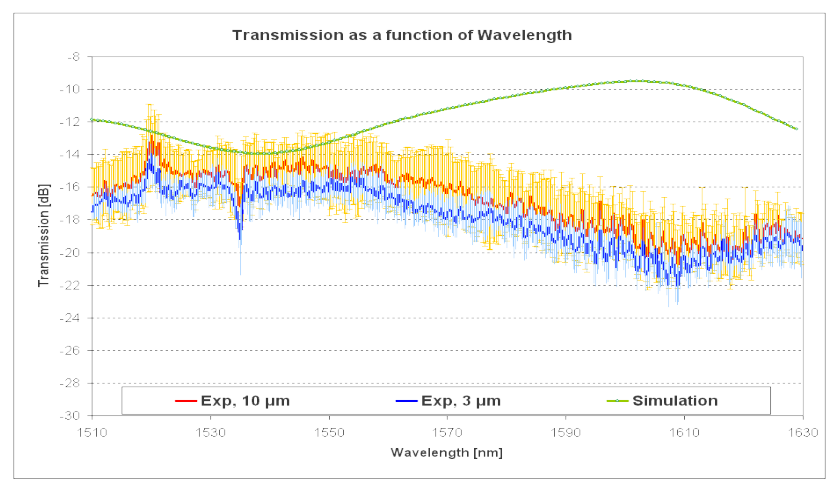

Fig. 1. Transmission as a function of wavelength, sensor with a $A u$-region length of $5 \mu \mathrm{m}$ 


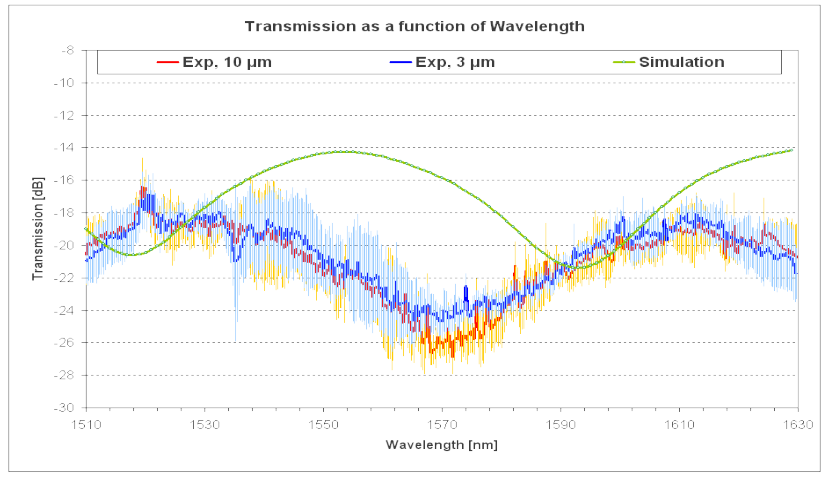

Fig. 2. Transmission as a function of wavelength, sensor with a $A u$-region length of $8 \mu \mathrm{m}$

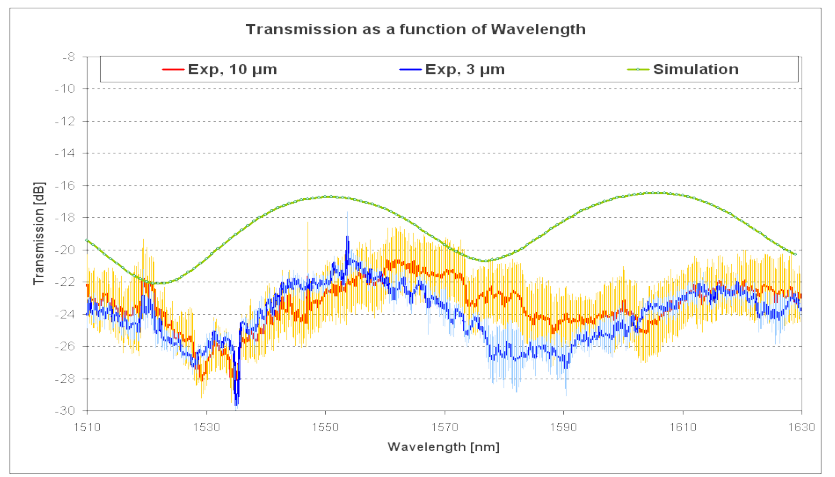

Fig. 3. Transmission as a function of wavelength, sensor with a $A u$-region length of $10 \mu \mathrm{m}$

While quantitative agreement between experiment and theory has not yet been obtained, one can deduce valuable information from the shape of these transmission spectra. The depth and width of the minima in transmission spectra of the experimental results and the theoretical predictions follow the same trend. For short sensor lengths (e.g. $5 \mu \mathrm{m}$ ) little contrast is expected, as the sensor length increases minima in transmission are more pronounced, the FWHM of these dips gets smaller and the Free-Spectral Range gets smaller as well.

\section{Bulk ReFractive Index SENSING}

To validate the prediction that this device can be used for sensing applications, we have characterized the change in transmission spectrum as a function of bulk refractive index. This was done by applying drops of liquids with varying refractive index above the sensor region with a $A u$-layer of length $8 \mu \mathrm{m}$. The example shown here 4 shows the change in transmission for a drop of pure DI-water versus a drop of an aqueous solution of $\mathrm{NaCl}$ (4 mass \%). While the measured blueshift is quite large $( \pm 19.5 \mathrm{~nm})$, further experiments need to be carried out to qualitatively characterize the sensitivity of this device.

\section{CONCLUSIONS}

We have presented in this paper a proof-of-principle for Surface Plasmon Interference sensors. Qualitative agreement

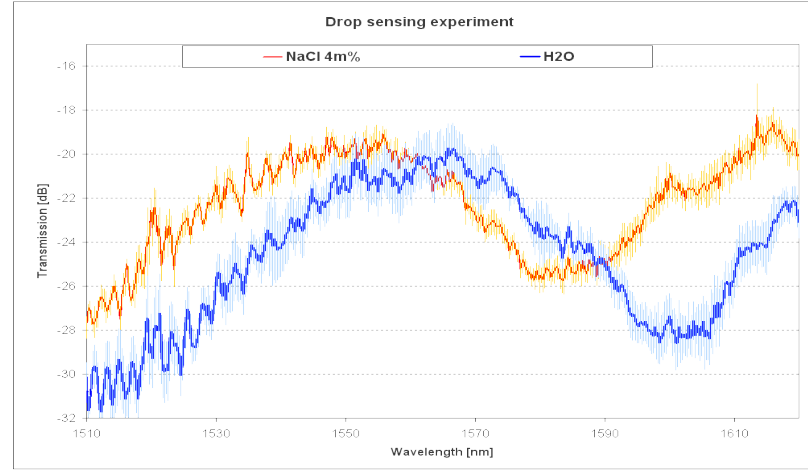

Fig. 4. Transmission as a function of wavelength for two distinct aqueous solution with different refractive indices. The large blue shift of the $\mathrm{NaCl} 4$ mass $\%$ indicates the potential of this device to be used as a biosensor.

between measurement results and theoretical data has been obtained. Furthermore, we have shown this device capable of detecting bulk refractive index changes, a first and indispensable step toward label-free biosensing. The measured blueshift is quite large, demonstrating the potential of this device to be used a a sensitive and label-free biosensor.

\section{ACKNOWLEDGMENT}

This work was carried out in the context of the GOA project (Ghent University) and was supported by the Belgian IAP PHOTON network. P. Debackere would like to thank S. Selvaraja for fabricating the a-Si samples.

\section{REFERENCES}

[1] Bienstman P and Baets R, Optical Modeling of Photonic Crystals and VCSELs using Eigenmode Expansion and Perfectly Matched Layers Opt. Quantum Electron 33(4-5):327-341 2001.

[2] Baets R, Taillaert D, Bogaerts W, Dumon P, De Vos K, Debackere P, Scheerlinck S, Van Thourhout D, Silicon Nanophotonics and its Applications in Sensing, CLEO, p. 1221-1222, 2007.

[3] Bogaerts W et al, Nanophotonic Waveguides in Silicon-on-Insulator Fabricated with CMOS Technology, Journal of Lightwave Technology, 23(1), p.401-412 2005.

[4] Debackere P, Bienstman P, Baets R , Improved ASR Convergence for the Simulation of Surface Plasmon Waveguide Modes, OWTNM 2006 Proceedings, p.14, 2006.

[5] Debackere P, Scheerlinck S,Bienstman P, Baets R, Surface Plasmon Interferometer, Optics Express, 14(16), p.7062-7072, 2007.

[6] Selvaraja S, Sleeckx E, Bogaerts W, Schaekers M, Dumon P, Van thourhout D and Baets R, Low loss amorphous silicon photonic wire and ring resonator fabricated by CMOS process, ECOC (PD),2007, Germany. 


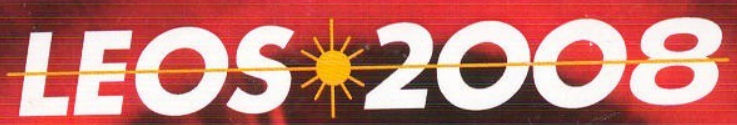

\section{IEEE LEOS Annual Meeting} Conference Proceedings

\section{9 - 13 November 2008 Marriot Newport Beach Hotel \& Spa Nerport Beach, CA}

